\title{
Transformer EMF in differential equations of synchronous generators
}

\author{
$L N$ Tokarev* \\ Department of Automated Control Systems, Saint Petersburg Electrotechnical University 'LETI', Saint Petersburg, Russia
}

\begin{abstract}
The article presents differential equations of a synchronous generator transformed according to the theory of two reactions, that is, in the axes d-q. We consider the influence of transformer EMF on transients. We prove that for the accurate calculation of transients in an electric power system it is necessary to use differential equations of the synchronous generator taking into account the transformer EMF in its stator.
\end{abstract}

\section{Introduction}

The results of the calculation of transients in electric power systems are an integral part of the design documentation for the electrical equipment of any vessel or ship. For more than half a century, technical standards have been used to calculate industry standards developed in Russia in the 1970s. The theoretical basis of these standards are differential equations of synchronous generators.

\section{Goals}

The goal of this article is to demonstrate the need to take into account the transformer EMF of a synchronous generator in the calculation of processes.

Until the beginning of the 1970s, the differential equations of a synchronous generator, presented below, were used to calculate transients in ship EPS.

$$
\begin{gathered}
U d=-r i d+w F q+p F d / w b ; \\
U q=-r i q+w F d-p F q / w b ; \\
U r=i r+\operatorname{Tr} p F r ; \\
0=i r d+\operatorname{Trd} p F r d ; \\
0=i r q+\operatorname{Trq} p F r q ; \\
F d=i r-x d i d+i r d ; \\
F q=x q i q+i r q ; \\
F r=i r-m d x d i d+g 1 \text { ird; } \\
\text { Frd }=i r d+g 2 i r-m d s x d i d ; \\
\text { Frq }=i r q+m q x q i q ; \\
\text { Me }=\text { Fd iq }+F q i d ;
\end{gathered}
$$

The equations above use the following notation:

$U, U q, U d$ are the stator voltage module of the synchronous generator and its components along the axes $\mathrm{d}$ and $\mathrm{q}$.

$i$, id, iq are the stator current module and its components along the axes,

$F d, F q$ are stator flux linkage components along the axes,

Frd, Frq are stator flux linkage components along the axes,

ird, irq are components of the damper current along the axes,

$w$ is rotor speed (in relative units),

$w b$ is base frequency (radian per second),

$\mathrm{Fr}$ is field winding flux linkage,

$u r$ is field winding voltage,

$i r$ is field current

$x d, x q$ are synchronous stator reactances

$x d s$ is transient reactance,

$x d 2 s, x q 2 s$ are subtransient reactances,

$m d, m q, m d s, g 1, g 2$ are winding magnetic coupling coefficients,

$M e$ is electromagnetic moment.

However, these equations are solved only in an approximate way. The easiest way is to simplify the stator equations by eliminating transformer EMFs. In order to obtain an exact solution to the differential equations of a synchronous generator, it is necessary to transform them in such a way that the stator voltage is a consequence of the presence of a generator excitation flux, and not vice versa.

At the end of the last century, the author found a transformation that made it possible to precisely solve the system of equations of the generator. In a transformed form, the system of equations of the synchronous generator is represented as follows:

$$
\begin{gathered}
p F r=(u r-i r) / T r ; \\
i r=F r+m d x d i d-g 1 i r d ;
\end{gathered}
$$




$$
\begin{gathered}
k f=(1-g 2) F r+(1-g 1) F r d ; \\
F d=Q k f-x d 2 s i d ; \\
F q=F r q+x q 2 s i q ; \\
k p f=(1-g 2) p F r+(1-g 1) p F r d ; \\
p F d=Q k p f+x d 2 s p i d, \\
p F q=p F r q+x q 2 s p i q . \\
Q=1 /(1-g 1 g 2) ; \\
i r d=Q(F r d-g 2 F r+m d s(1-g 1) x d i d) ; \\
p F r d=-\operatorname{Ird} / \operatorname{Trd} ; \\
i r q=F r q-m q x q i q ; \\
p F r q=-i r q / T r q ; \\
U q=-r i q+w F d-p F q / w b ; \\
U d=-r i d+w F q+p F d / w b ;
\end{gathered}
$$

Here, we denominate as $\boldsymbol{k} \boldsymbol{f}$ and $\boldsymbol{p} \boldsymbol{k} \boldsymbol{f}$ the parts of the equations that are selected to reduce the length of the lines. From the transformed system of equations it follows that the stator voltage is an output variable, as it is in the physical system.

We perform the calculation of transients in the electric power system according to the converted equations, that is, taking into account the transformer EMF. A listing of the processes of turning on and off the load of a synchronous generator, as well as a short circuit is shown in the figure.

Let us see, for comparison, what happens if the calculation program of, for example, voltage changes during switching the load on and off, as well as short circuit currents, is compiled using simplified equations, excluding transformer EMFs ( $p F d$ and $p F q$ ) from them.

As can be seen from the printout shown in Figure 2, the simplification of the equations particularly significantly affected the duration of the process of changing the short circuit current. The duration of the process is reduced by more than an order of magnitude. Therefore, the calculation, for example, of the Joule integral, necessary for evaluating the thermal stability of a switching device in a short-circuited circuit, will give a significantly erroneous result. The values of short-circuit shock current and voltage changes when switching on and off the rated load differ as well.

The transient curves obtained using the simplified equations are similar in appearance to the curves presented in Figure 1. However, the numerical values of the variables are different. To illustrate it, Figure 2 shows the transient curves obtained by simplified equations.

For a more detailed discussion of processes in the short-circuit mode, we change the time scale for printing the results and leave only the voltage and current of the generator stator. Let us first consider the calculation result obtained by the complete (not simplified) equations.

As we can see, the damping time of the process of changing the short circuit current is about $0.4 \mathrm{~s}$. The rate of change of the current curve is $50 \mathrm{~Hz}$. The voltage of the generator at the time of a short circuit decreases almost immediately to a very small value.

Let us consider a printout of a process calculated

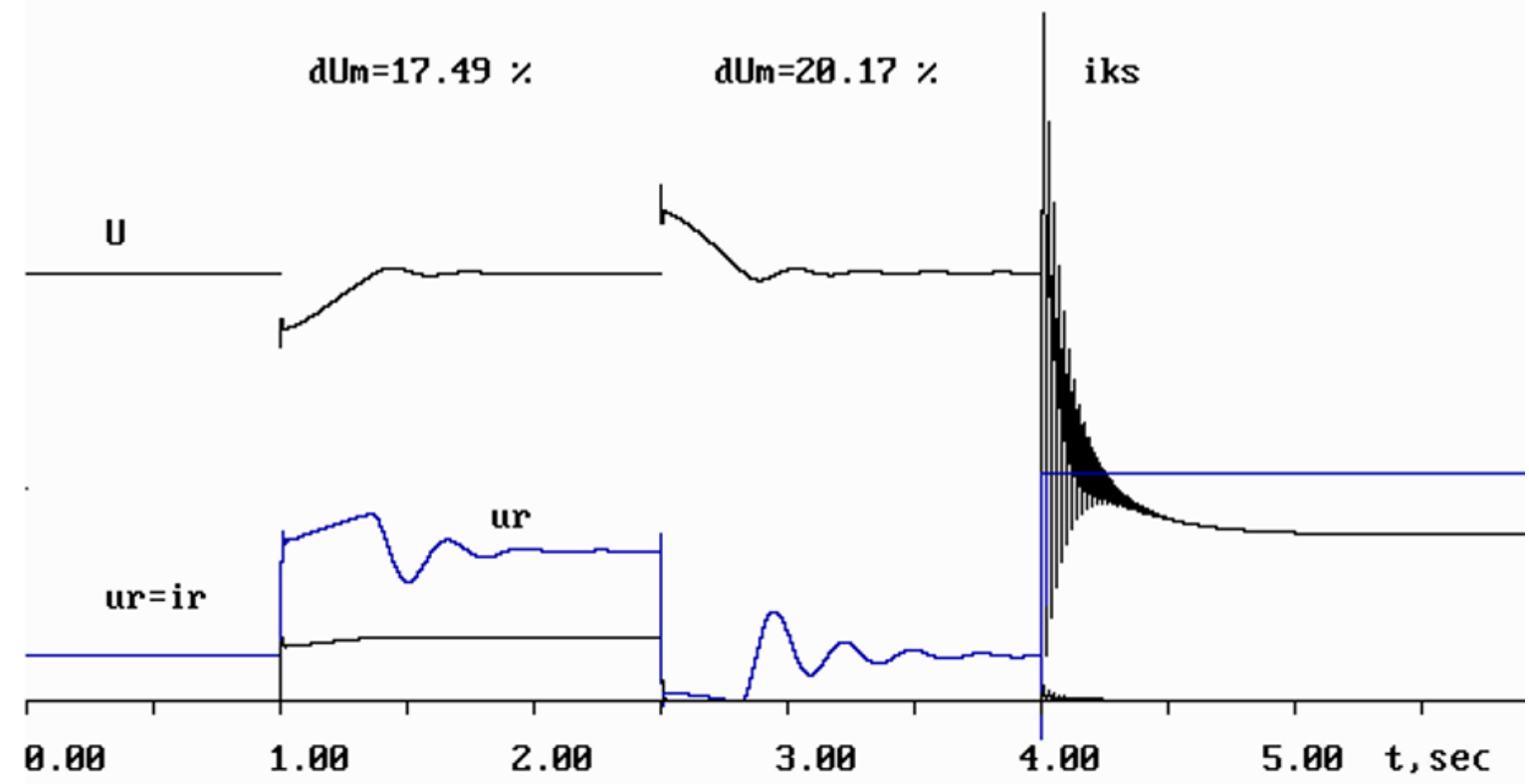

Fig. 1. Printout of the results of the calculation of transients in the electric power system, obtained according to the complete equations, in which the transformer EMF of the synchronous generator is not excluded. $u r$ and $i r$ are the voltage and current of the rotor, $i$ is the stator current, $i k s$ is the short circuit current, $d U m$ is the voltage change when the load is turned on and off, $t$ is the time. 


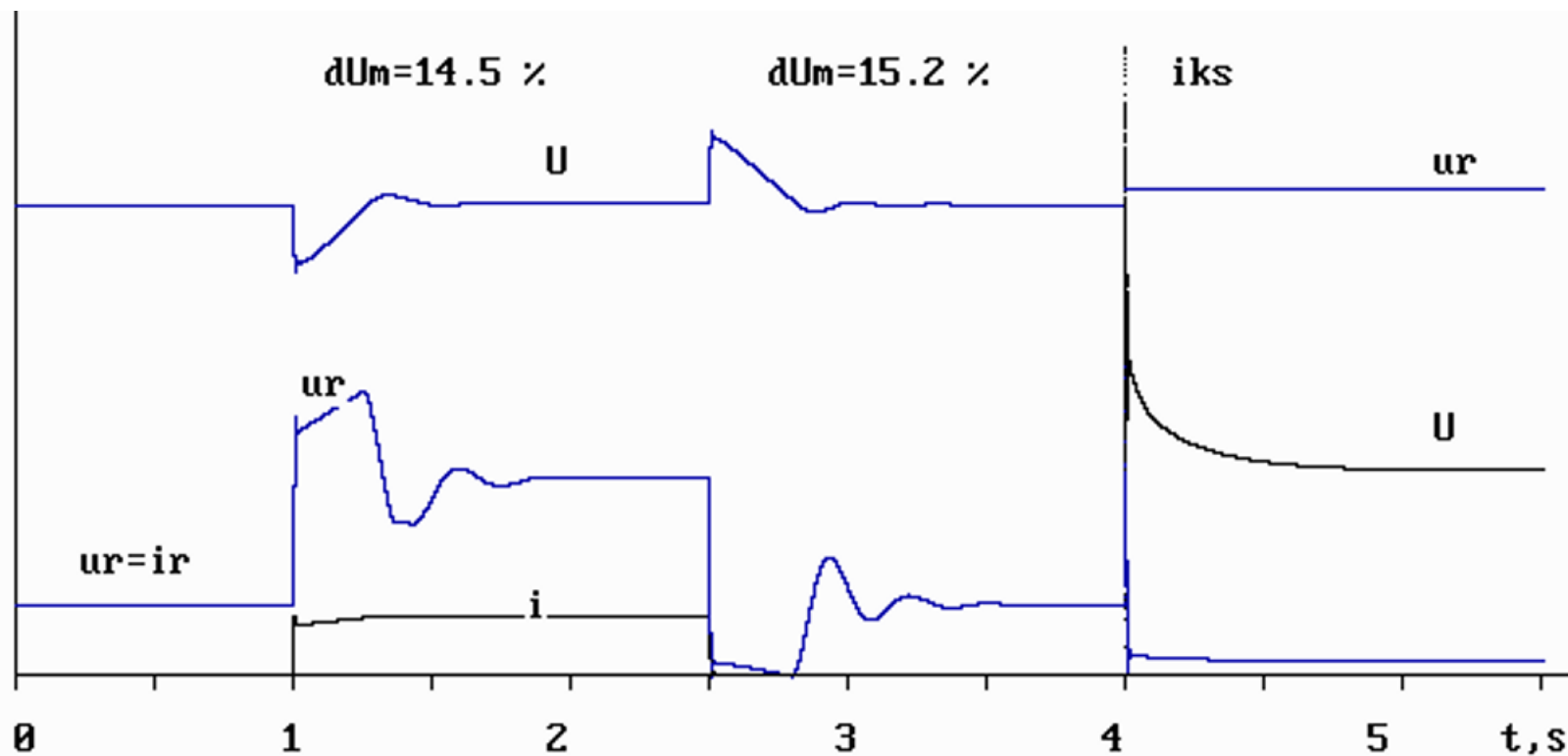

Fig. 2. Printout of the results of the calculation of transients in the electric power system, obtained according to simplified equations, in which the transformer EMF of the synchronous generator is excluded. $u$ is the stator voltage, $u r$ and $i r$ are the rotor voltage and current, $i$ is the stator current, $i k s$ is the short circuit current, $d U m$ is the voltage change when the load is turned on and off, $t$ is the time.

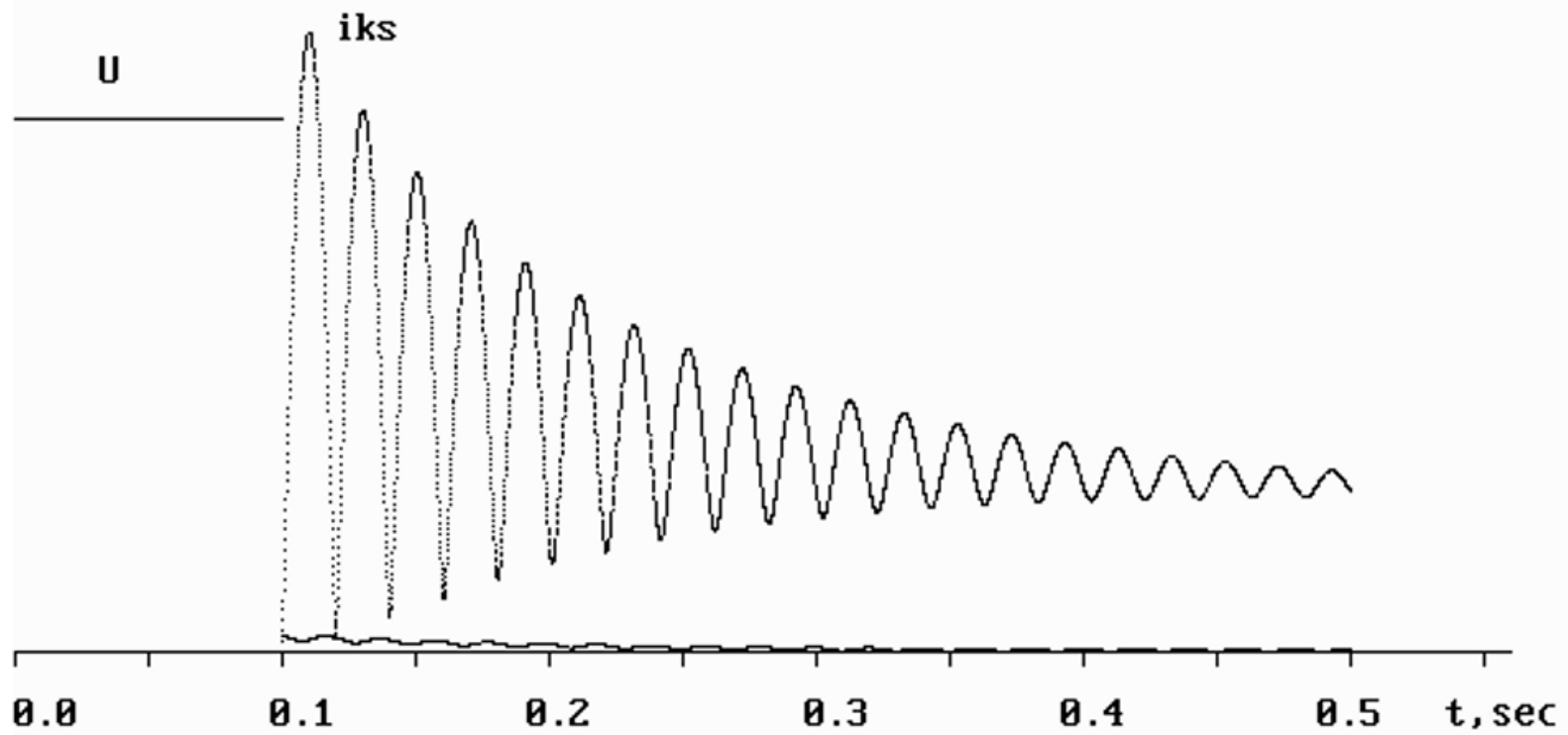

Fig. 3. Printout of the results of the calculation of transients during a short circuit in the electric power system, obtained according to the complete equations, in which transformer EMFs of the synchronous generator are not excluded. $u$ is the stator voltage, $i k s$ is the short circuit current, $t$ is the time.

using simplified equations in which transformer EMFs of a synchronous generator are excluded. The process is shown in Figure 4.

As we can see, the duration of the process of changing the short circuit current is about $0.01 \mathrm{~s}$, which is 40 times less than when calculating according to complete equations, taking into account transformer EMF. The frequency of current changes is sharply increased and amounts to two kilohertz. The voltage curve is very different from the one shown in Figure 3.

\section{Results}

Obviously, the value of the Joule integral, which is used to evaluate the thermal stability of the switching protective device, which disconnects the short circuit current, when using simplified equations for calculating, will be estimated with an unacceptable error. 


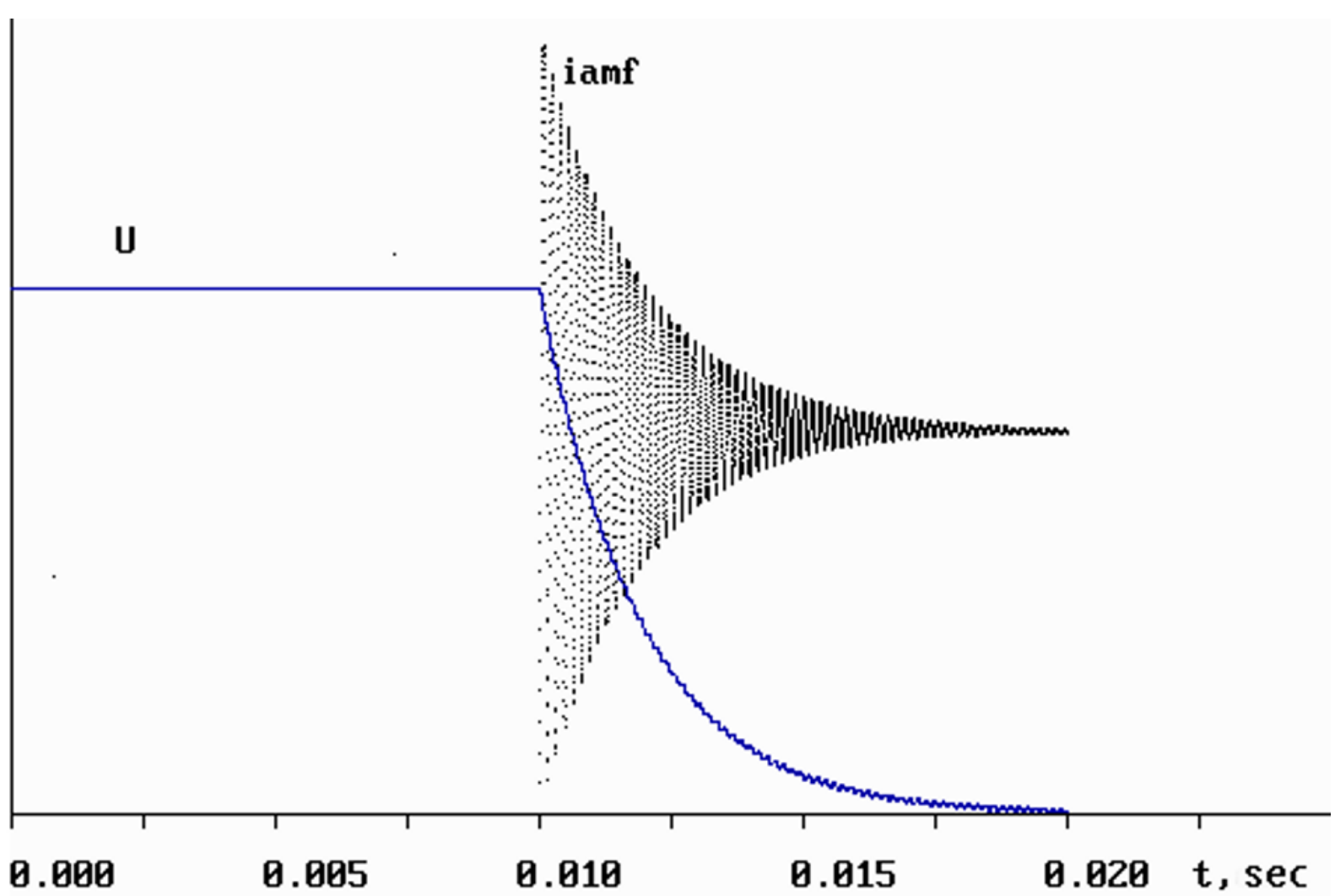

Fig. 2. Printout of the results of the calculation of transients during a short circuit in the electric power system, obtained according to simplified equations in which transformer EMFs of a synchronous generator are excluded. $u$ is the stator voltage, $i k s$ is the short circuit current, $\mathrm{t}$ is the time.

\section{Conclusion}

For an accurate calculation of transients in the electric power system, it is necessary to use the differential equations of the synchronous generator that take into account the transformer EMF in the stator.

\section{References}

1. P.H. Park, Theory of two reactions of synchronous machines, 48(1) (1929)

2. A.A. Gorev, Transients of a synchronous machine (1950)

3. L.N. Tokarev, O.N. Klimanov, V.N. Tolcheev, Calculation of voltage and currents of a synchronous generator using Gorev-Park equations (1968)

4. L.N. Tokarev, Mathematical description, calculation and modeling of physical processes in ship power plants (1980) 